\title{
Spectroscopic Properties of Light Baryon
}

\author{
Chandni Menapara* and Ajay Kumar Rai \\ Department of Physics, Sardar Vallabhbhai National Institute of Technology, Surat-395007, Gujarat, \\ India.
}

\begin{abstract}
Hadron Spectroscopy provides a realm to study the internal quark dynamics within the hadrons through phenomenological, theoretical as well as experimental approaches. In the present article, an attempt has been made to exploit the nucleon $\mathrm{N}$ resonances using a non-relativistic hypercentral Constituent Quark Model (hCQM). The properties are studied based on the linear nature of confining part of the potential. The $1 \mathrm{~S}-5 \mathrm{~S}, 1 \mathrm{P}-3 \mathrm{P}, 1 \mathrm{D}-2 \mathrm{D}$ and $1 \mathrm{~F}$ states mostly with four star labelled resonances are explored again with the separation of charge states using different constituent quark masses. Also, Regge trajectories for some obtained states are plotted for examining the linear nature.
\end{abstract}

\section{Introduction}

Hadronic Physics aims at understanding the hadrons as bound state of quarks and gluons interaction alongwith the excited spectrum. Baryon spectroscopy particularly attempts to study the resonance states of all the possible spin-parity as well as exploring other properties like magnetic moment, decay, etc [1]. Even after the knowledge of confinement and asymptotic freedom, the strong interaction still poses different challenges and puzzles. Experimental facilities worldwide have tried to produce hadrons through hadronization process in order to reveal yet hidden properties. The light sector of baryons even after so many years of findings still provide us with challenges to certainly understand the interaction inside the composite systems. Also, the decay of many heavy unstable baryons lead to the production of many light, strange baryons in the output.

The $\mathrm{N}$ baryons with $\mathrm{u}$ and $\mathrm{d}$ quark combinations with mixed symmetry wavefunction have been observed experimentally with large number of established resonances [2]. $I=\frac{1}{2} P\left(\right.$ uud, $\left.I_{3}=\frac{1}{2}\right), N\left(\right.$ udd, $\left.I_{3}=-\frac{1}{2}\right)$ with $S=\frac{1}{2}$

$\mathrm{N}$ and $\Delta \mathrm{s}$ have been studied through photoproduction decays by ELSA[3]. $\mathrm{N}$ has been at focus of the facilties at JLab, CLAS, MAMI [4, 5] and others. The upcoming facilities like PANDA at FAIR-GSI is highly aimed for light, strange baryon studies [6, 7]. Theoretical and phenomenological approaches study $\mathrm{N}$ such as Isgur-Karl model [8] Goldstone-boson exchange model [10], relativistic approach [9], quark-diquark system along with GurseyRadicati exchange intercation [11,12]. Lately, varied approaches based on QCD SUM Rules [16], basis light-front model [17] and light-front relativistic [18], Lattice QCD [19] and covariant Faddeev approach [20] and others based on $\mathrm{n}$ and $J^{P}$ values and trajectories.

\footnotetext{
*e-mail: chandni.menaparagmail.com
} 


\section{[21, 23], Skyrme model, AdS/QCD, etc.}

In the present article, an attempt has been made to apply hypercentral Constituent Quark Model (hCQM) for both the isospin states of $\mathrm{N}$ which is based on varied the ground state $1 \mathrm{~S}$. The results have been compared with other models as well as our earlier work where the same 1S state parameters i.e. $\mathrm{u}$ and d considered with same constituent mass. Also, later Regge trajectory has been plotted for some states for the reference of linear nature of $\left(\mathrm{n}, M^{2}\right)$ and $\left(\mathrm{J}, M^{2}\right)$.

\section{Theoretical Formalism}

The non-relativistic formalism is used to explore the resonance states of baryons. The hypercentral Constituent Quark Model (hCQM) is based on parametrizing all the effects within a baryon in the form of constituent quark masses [24]. The three body interaction is taken care of using Jacobi coordinates, ultimately reducing to hyperradius $\mathrm{x}$ and hyperangle $\xi$. The potential consisting of a Coulomb-like part and another confining part depends solely on the hyperradius $\mathrm{x}$ here. Jacobi coordinates takes care of the three body dynamics as given below.

$$
\begin{gathered}
\rho=\frac{1}{\sqrt{2}}\left(\mathbf{r}_{1}-\mathbf{r}_{2}\right) ; \lambda=\frac{1}{\sqrt{6}}\left(\mathbf{r}_{1}+\mathbf{r}_{2}-2 \mathbf{r}_{3}\right) \\
x=\sqrt{\rho^{2}+\lambda^{2}} ; \quad \xi=\arctan \left(\frac{\rho}{\lambda}\right)
\end{gathered}
$$

The Hamiltonian appears to be,

$$
H=\frac{P^{2}}{2 m}+V(x)+V_{S D}(x)+V^{1}(x)
$$

Thus the hyper-radial part of the wave-function as determined by hypercentral Schrodinger equation is

$$
\left[\frac{d^{2}}{d x^{2}}+\frac{5}{x} \frac{d}{d x}-\frac{\gamma(\gamma+4)}{x^{2}}\right] \psi(x)=-2 m[E-V(x)] \psi(x)
$$

The confinement realm of the potential is taken to be of linear nature as earlier explored in various studies.

$$
V(x)=-\frac{\tau}{x}+\alpha x
$$

Also, the splitting of a given state into all possible angular momentum quantum number $\mathrm{J}$ has been considered by adding the spin-dependent terms to the above potential,

$$
V_{S D}(x)=V_{S S}(x)\left(\mathbf{S}_{\rho} \cdot \mathbf{S}_{\lambda}\right)+V_{\gamma S}(x)(\gamma \cdot \mathbf{S})+V_{T} \times\left[S^{2}-\frac{3(\mathbf{S} \cdot \mathbf{x})(\mathbf{S} \cdot \mathbf{x})}{x^{2}}\right]
$$

Here, $V_{S S}(x), V_{\gamma S}(x)$ and $V_{T}(x)$ are spin-spin, spin-orbit and tensor terms respectively [25].

To further refining the resonance masses, a first order correction term with $\frac{1}{m}$ dependence has been included.

$$
V^{1}(x)=-C_{F} C_{A} \frac{\alpha_{s}^{2}}{4 x^{2}}
$$

where $C_{F}$ and $C_{A}$ are Casimir elements of fundamental and adjoint representation. $\alpha_{s}$ is the running coupling constant. The statis potential with leading order contribution followed by relativistic corrections in the power of $\frac{1}{m}$ term has been noted for quarkonia systems [26]. 
Also, similar study has been undertaken for heavy-light systems as well [27] which has been a key to include the correction term for light systems.

The Schrodinger equation with the hyper-radial part is numerically solved for calculating the excited state masses [28]. Details of the model can be found in our other articles [31-36].

\section{Results and Discussions}

Using the above potential model, the masses are computed for $1 \mathrm{~S}-5 \mathrm{~S}, 1 \mathrm{P}-3 \mathrm{P}, 1 \mathrm{D}-2 \mathrm{D}$ and $1 \mathrm{~F}$ states. Earlier $N^{*}$ resonances have been studied on the footings of the same constituent quark mass i.e. $0.290 \mathrm{GeV}$ for both $\mathrm{u}$ and d quarks, employing same value for $1 \mathrm{~S}$ state for all the members [32]. However, current study segregates all the isospin partners using the separate constituent quark mass as $m_{u}=0.290$ and $m_{d}=0.300$. The excited states are recalculated for both the isospin states and compared with various results in the table [1] below.

The ground state parameters lead to the value of $938 \mathrm{MeV}$ for $\mathrm{P}$ and $948 \mathrm{MeV}$ for $\mathrm{N}$, whereas earlier both the masses were $939 \mathrm{MeV}$ such that excited states cannot be separated out. The next four star status $\mathrm{N}(1440)$ with $J^{P}=\frac{1}{2}^{+}$is the $2 \mathrm{~S}$ state and present results 1419 and 1427 are well within the PDG range and other approaches too. Similarly the $3 \mathrm{~S}$ and $4 \mathrm{~S}$ states are found to be with good agreement with experimental results.

The very first state in negative parity is $\mathrm{N}(1520)$ with $J^{P}=\frac{3}{2}^{-}$is reproduced as 1494 and 1502 respectively for $\mathrm{P}$ and $\mathrm{N}$. Also, this state is lower than its spin partner i.e. $J^{P}=\frac{1}{2}^{-}$ $\mathrm{N}(1535)$ is also consistent with our results as the model is predicting lower mass for higher spin state. Here, it is noteworthy that $\mathrm{N}(1650) J^{P}=\frac{1}{2}^{-}$doesn't appear in the present dat as the $\frac{5}{2}^{-}$is just $25 \mathrm{MeV}$ away which falls under 1P partners. In case of 2P states, PDG as well as results from BGR collaboration [30] are compared.

$\mathrm{N}(1720) \frac{3}{2}^{+}$with four star label is in coherence with most of the references given in comparison. $1 \frac{1}{2}^{+}$appears as a higher state than $\frac{3}{2}^{+}$and $\frac{5}{2}^{+}$. Also, states in $2 \mathrm{D}$ are found to vary within $100 \mathrm{MeV}$ difference compared to all.

All the negative parity states of $1 \mathrm{~F}$ have been observed and assigned three and four star status. In the present results, higher spin state with $J^{P}=\frac{9}{2}^{-}$is slightly under-predicted compared to other results and PDG. The two states which doesn't appear in earlier study have been calculated here. The $\mathrm{N}(2220) 1 \mathrm{G} \frac{9}{2}^{+}$obtained to be 2371 and 2376 respectively which is differing by $70 \mathrm{MeV}$ from PDG range. Also, the $\mathrm{N}(2600)$ is assigned $1 \mathrm{H} \frac{11}{2}^{-}$is very well reproduced in the current results.

The other models mentioned for the comparison include relativistic interacting quarkdiquark model with Gursey Radicati-inspired exchange interaction [12]. The another very recent study of mass spectra of all light strange baryon is given by N. Amiri et. al. using Bethe Ansatz introducing U(7) algebra [13]. A. V. Anisovich et. al. has reproduced the $\mathrm{N}$ and $\Delta$ spectrum using the multichannel partial wave analysis of pion and photo-induced reactions [14]. The later are quark model using hyperfine interactions due to two-gluon exchange between quarks [22], semi-relativistic constituent quark model [15], chiral quark model [29], mass formula [23] and dynamical chirally improved quarks [30]. 
Table 1: Masses of $\mathrm{P}$ and $\mathrm{N}$ Baryons (in $\mathrm{MeV}$ )

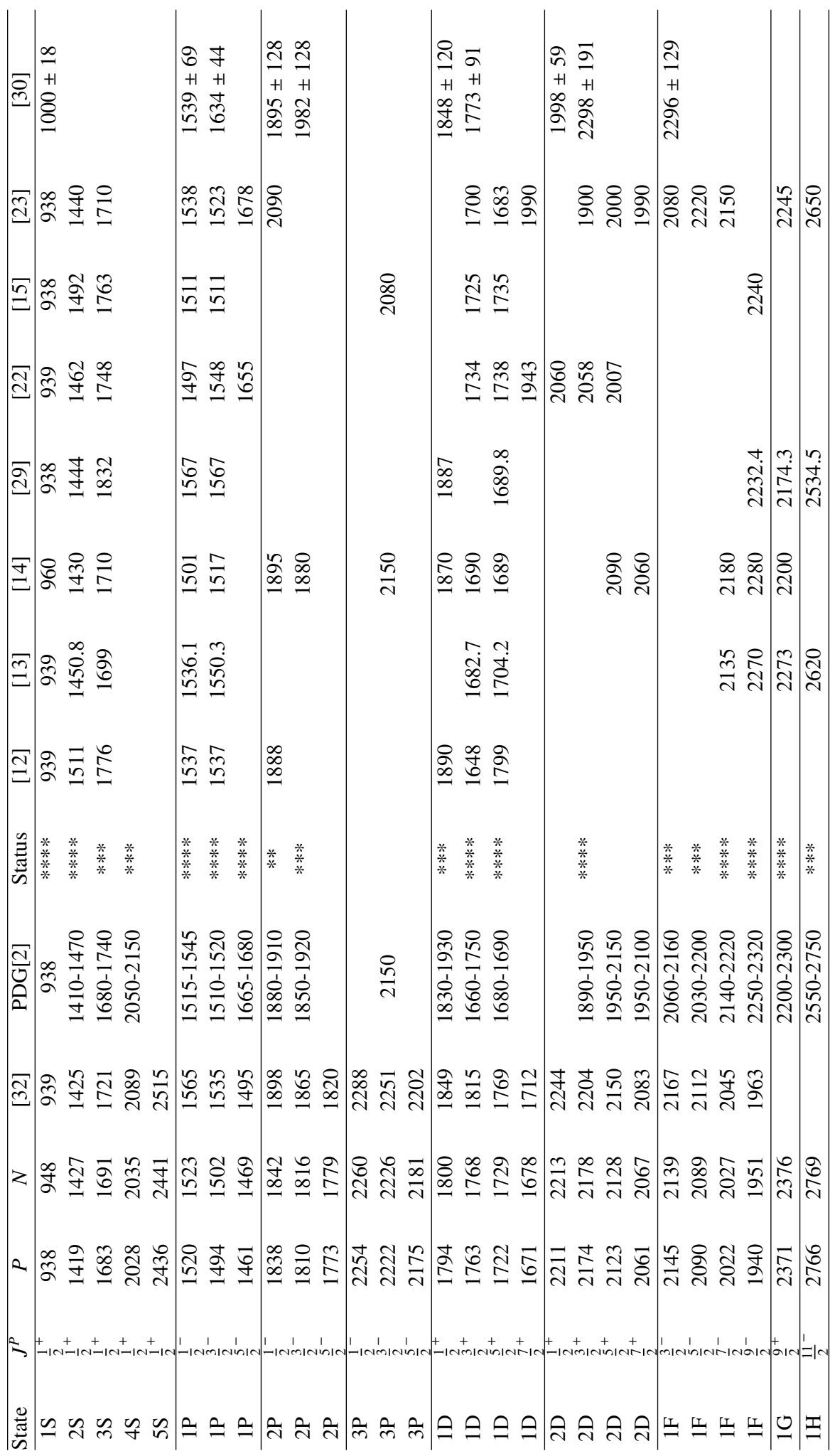




\section{Regge Trajectory}

Regge trajectories have been one of the useful tools in spectroscopic studies. The plot of total angular momentum $\mathrm{J}$ and principle quantum number $\mathrm{n}$ against the square of resonance mass $M^{2}$ have been in the form of linear equation.

$$
\begin{aligned}
& J=a M^{2}+a_{0} \\
& n=b M^{2}+b_{0}
\end{aligned}
$$

The non-intersecting and linearly fitted lines have been in accordance with theoretical and experimental data in many studies. These plots might be helpful in predicting the correct spin-parity assignment of a given state. For the present data, we have plotted $\left(\mathrm{n}, M^{2}\right)$ and $\left(\mathrm{J}, M^{2}\right)$ with natural parity and have been found to follow the linear trend.

Figure 1. Regge trajectory $N$ for $n \rightarrow M^{2}$

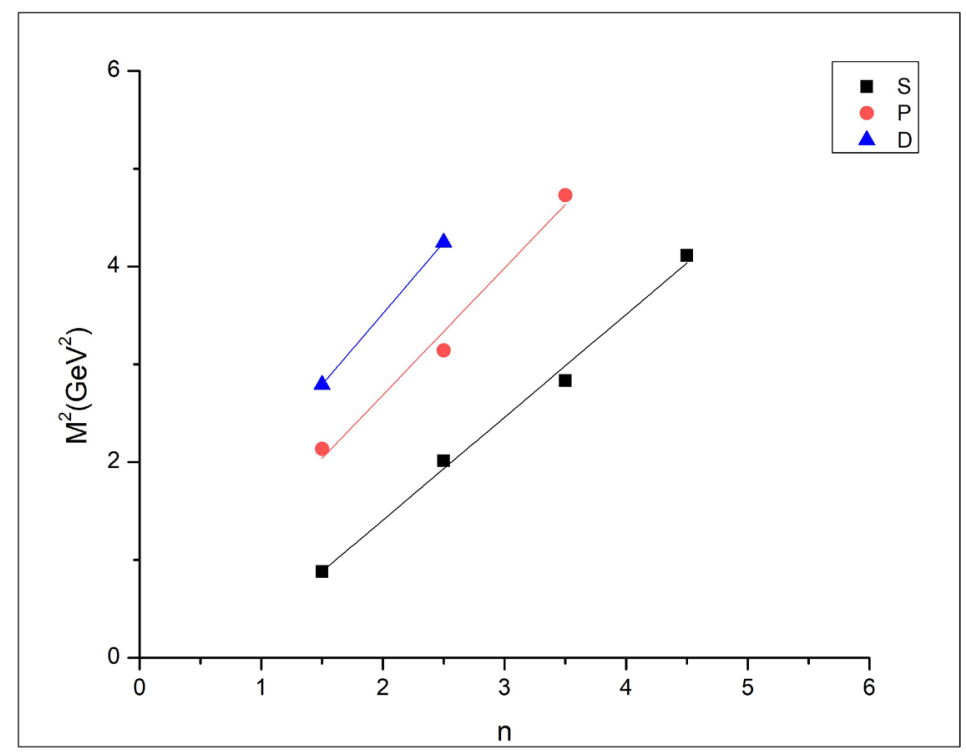

\section{Magnetic Moment}

Baryon magnetic moment and transition magnetic moment plays a crucial role in providing information regarding the structure and shape of baryon. Magnetic moment shall have contribution from many other effects within the baryon as sea quark, valence quark, orbital etc [37]. Various models have contributed to obtaining the octet and decuplet baryons' magnetic moment $[38,39]$.

$$
\mu_{B}=\sum_{q}\left\langle\phi_{s f}\left|\mu_{q z}\right| \phi_{s f}\right\rangle
$$

where $\phi_{s f}$ is the spin-flavour wave function.

$$
\mu_{q z}=\frac{e_{q}}{2 m_{q}^{e f f}} \sigma_{q z}
$$


Figure 2. Regge trajectory $N$ for $J \rightarrow M^{2}$

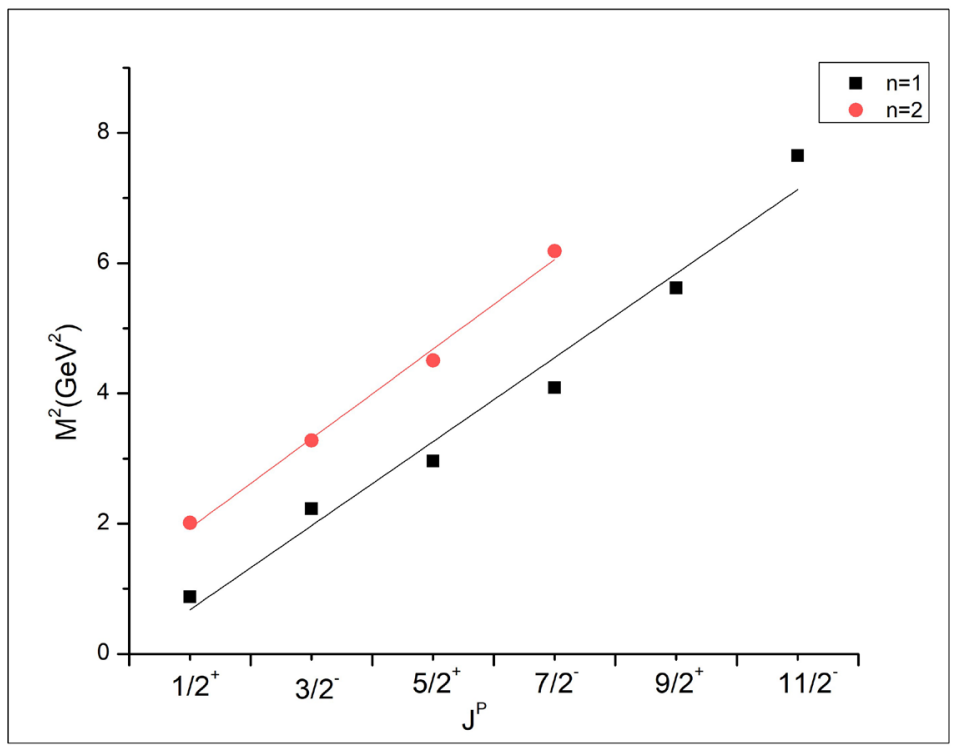

The effective mass of quark $m_{q}^{e f f}$ is calculated using

$$
m_{q}^{e f f}=m_{q}\left(1+\frac{\langle H\rangle}{\sum_{q} m_{q}}\right)
$$

Using the above equations, magnetic moment have been calculated for $\mathrm{P}$ and $\mathrm{N}$ with effective quark mass, other than the constituent quark mass. For proton, $\mu_{B}=3.03 \mu_{N}$ as compared to $2.79 \mu_{N}$ experimental value. For $\mathrm{N}, \mu_{B}=-1.97 \mu_{N}$ compared to $-1.91 \mu_{N}$ experimental value.

\section{Conclusion}

The present study has employed a non-relativistic, hypercentral Constituent Quark model (hCQM) with potential with linear term, spin-dependent terms as well as first order correction term. It has been attempted to segregate the isospin states of nucleons by introducing a different constituent quark mass for $\mathrm{u}$ and d quarks which provided with $938 \mathrm{MeV}$ and 948 $\mathrm{MeV}$ respectively. Similarly all the excited states are reproduced for both and comparison shows an overall good agreement with experimental results and various approaches. One state each for $1 \mathrm{G}$ and $1 \mathrm{H}$ has been obtained as well. The Regge trajectories show a well fitted linear curves for (n, $\left.M^{2}\right)$ and $\left(\mathrm{J}, M^{2}\right)$ where natural parities upto $\frac{9}{2}$ and $\frac{11}{2}$ are included. In addition, the magnetic moment values have been revised for $\mathrm{P}$ and $\mathrm{N}$ using the effective quark mass which are quite similar to PDG values.

\section{Acknowledgement}

Ms. Chandni Menapara would like to acknowledge the support from the Department of Science and Technology (DST) under INSPIRE-FELLOWSHIP scheme for pursuing this work.

Authors are thankful to the organisers of a Virtual Tribute to Quark Confinement and Hadron Spectrum, 2021 for giving us the opportunity to participate and present our work. 


\section{References}

[1] V. Crede and W. Roberts, Rept. Prog. Phys., 76: 076301 (2013), arXiv:1302.7299 [hep$\mathrm{ph}]$

[2] P.A. Zyla et al., (Particle Data Group), Prog. Theor. Exp. Phys. 2020, 083C01 (2020) and 2021 update

[3] U. Thoma et al. (CB-ELSA Collaboration), (2007), arXiv: 0707.3592v4 [hep-ph]

[4] I. G. Aznauryan et al, Int. J. Mod. Phys. E, 221330015 (2013), arXiv:1212.4891 [hep$\mathrm{ph}]$

[5] V. I. Mokeev, V. D. Burkert, D. S. Carman, L. Elouadrhiri et al, Phys. Rev. C, 93025206 (2016)

[6] B. Singh et al. [PANDA Collaboration], J. Phys. G: Nucl. Part. Phys. 46, 045001 (2019), Phys. Rev. D 95, 3, 032003 (2017), Eur. Phys. J. A 52 10, 325 (2016); Eur. Phys. J. A 51, 107 (2015); Nucl. Phys. A 954, 323-340 (2016)

[7] G. Barruca at al., [PANDA Collaboration], G. Barucca et al. (PANDA Collaboration), Eur. Phys. J. A 57, 1-36 (2021); Eur. Phys. J. A 57, 149 (2021); Eur. Phys. J. A 57, 1-22 (2021); Eur. Phys. J. A 55, 42 (2019);

[8] N. Isgur and G. Karl, Phys. Rev. D. 18, 4187 (1978)

[9] S. Capstick and N. Isgur, Phys. Rev. D 34, 2809 (1986)

[10] L. Ya. Glozman, Z. Papp and W. Plessas, Phys. Lett. B 381, 311 (1996)

[11] E. Santopinto, Phys. Rev. C 72, 022201(R) (2005)

[12] E. Santopinto and J. Ferretti, Phys. Rev. C 92, 025202 (2015)

[13] N. Amiri, M. Ghapanvari and M. A. Jafarizadeh, Eur. Phys. J. Plus 141, 136 (2021)

[14] A.V. Anisovich, R. Beck, E. Klempt, V.A. Nikonov, A.V. Sarantsev and U. Thoma, Eur. Phys. J. A 48, 15 (2012)

[15] M. Aslanzadeh and A. A. Rajabi, Int.J.Mod.Phys. E26, 1750042 (2017)

[16] K. Azizi, Y. Sarac and H. Sundu, J.Phys.G 47, 9, 095005 (2020) arXiv:1909.03323v2 [hep-ph]

[17] J.P.Vary et al., Few Body Syst. 59, 56 (2018), arXiv:1804.07865 [nucl-th]

[18] I. G. Aznauryan and V. D. Burkert, Phys. Rev. C 85, 055202 (2012), arXiv:1201.5759 [hep-ph]

[19] R. G. Edwards, J. J. Dudek, D. G. Richards, and S. J. Wallace Phys. Rev. D 84, 074508 (2011)

[20] H. Sanchis-Alepuz and C. S. Fischer, Phys. Rev. D 90, no. 9, 096001 (2014)

[21] Y. Chen, B.Q. Ma, Chin. Phys. Lett. 25, 3920 (2008)

[22] Y. Chen, B.-Q. Ma, Nucl. Phys. A 831, 1 (2009)

[23] E. Klempt, arXiv:nucl-ex/0203002 (2002)

[24] M. M. Giannini, E. Santopinto, Chin. J. Phys., 53, 020301 (2015)

[25] M. B. Voloshin, Prog. Part. Nucl. Phys. 61, 455-511 (2008)

[26] Y. Koma, M. Koma and H. Wittig, Phys. Rev. Lett. 97, 122003 (2006)

[27] Z. Shah, K. Thakkar, A. K. Rai and P. C. Vinodkumar, Eur. Phys. J. A 52313 (2016); Chin. Phys. C 40, 12, 123102 (2016)

[28] W. Lucha, F. schoberls, Int. J. Modern Phys. C. 10607 (1997)

[29] Z. Ghalenovi and M. Moazzen, Eur. Phys. J. Plus 132, 354 (2017)

[30] G. P. Engel, C. B. Lang, D. Mohler and A. Schaefer [BGR Collaboration] Phys. Rev. D. 87, 074504 (2013)

[31] K. Gandhi and A. K. Rai, Eur. Phys. J. Plus, 135, 213 (2020) 
[32] Z. Shah, K. Gandhi and A. K. Rai, Chin. Phys. C 43, 034102 (2019), arXiv:1812.04858 [hep-ph]

[33] C. Menapara, Z. Shah and A. K. Rai, Chin. Phys. C 45, 023102 (2021)

[34] C. Menapara and A. K. Rai, Chin. Phys. C 45, 063108 (2021)

[35] C. Menapara, Z. Shah and A. K. Rai, AIP Conf.Proc. 2220, 140014 (2020)

[36] C. Menapara and A. K. Rai, DAE Symp.Nucl.Phys. 64 673-674 (2020)

[37] H. Dahiya and M. Gupta, Phys. Rev. D 67, (2003) 114015

[38] K. Thakkar, B. Patel, A. Majethiya and P. C. Vinodkumar, PRAMANA J of Physics 77, (2011) 1053-1067

[39] Fayyazuddin and M. J. Aslam, arXiv:2011.06750 [hep-ph] (2020) 